(C) The Author(s)

DOI: $10.1142 / \mathrm{S} 2010194518601175$

\title{
Investigating artifacts associated with $\beta$-particle interactions in charge-coupled devices
}

\author{
Rosie Newton \\ Engineering Department, Lancaster University \\ Lancaster, LA1 4YW, UK \\ r.newton1@lancaster.ac.uk \\ Mike J. Scott \\ BIC Technology Ltd., Gostin House, 32/36 Hanover St. \\ Liverpool, $L 14 L N$, UK \\ Malcolm J. Joyce \\ Engineering Department, Lancaster University \\ Lancaster, LA1 4YW, UK \\ m.joyce@lancaster.ac.uk
}

Published 10 July 2018

\begin{abstract}
Charge-coupled devices (CCDs) show potential for detecting charged particles and ionizing radiation. In particular, the clusters in the pixel images produced can be distinctive for $\alpha$ and $\beta$ radiation, with $\alpha$ particles causing symmetrical clusters or vertical tracks, and $\beta$ particles causing long, curved tracks. This distinction may be exploited by means of a handheld, portable device for in-situ detection, and identification of radioactive contamination. $\beta$-particle track interactions in CCDs have been investigated. Simulative results using CASINO (Monte Carlo Simulation of Electron Trajectory in Solids) attempt to predict the size of $\beta$-particle pixel clusters, using $512 \mathrm{keV}$ and $310 \mathrm{keV}$ electrons to represent ${ }^{137} \mathrm{Cs}$ and ${ }^{60} \mathrm{Co}$, respectively. The number of pixels that higherenergy electrons traversed peaked at two, while lower-energy electrons had a smaller peak of 2.5 pixels, with a higher proportion of large cluster sizes. This finding is consistent with the higher scattering cross-section for lower-energy $\beta$ particles. By contrast, experimental data show a peak at one pixel for both sources, owing to the addition of smaller $\gamma$ clusters. The ${ }^{60} \mathrm{Co}$ source shows a higher proportion of large cluster sizes than the ${ }^{137} \mathrm{Cs}$, as was also seen in the simulation; however, the difference was small, as these sources are similar in energy. Simulative and experimental data will be used to process the CCD images further, with the objective of distinguishing between $\beta$ and $\gamma$ radiation. Investigations have also been carried out using a ${ }^{210} \mathrm{Po} \alpha$ particle source. Horizontal streaks were seen in the images produced, with an average length of 14 pixels. Further research will be performed using an accelerator to obtain different $\alpha$-particle energies.
\end{abstract}

Keywords: Alpha spectroscopy; beta spectroscopy; charge-coupled device.

This is an Open Access article published by World Scientific Publishing Company. It is distributed under the terms of the Creative Commons Attribution 4.0 (CC-BY) License. Further distribution of this work is permitted, provided the original work is properly cited. 


\section{Introduction}

Detecting and identifying radioactive contamination is essential in the nuclear industry but may be difficult to achieve in situ for $\alpha$ radiation, owing to its highly ionizing nature. Because $\alpha$ radiation is extremely harmful if inhaled or ingested, identifying $\alpha$ contamination is critical. Usually, $\alpha$-particle spectroscopy is performed in a laboratory by processing the sample to isolate the radioisotope and detecting the $\alpha$ radiation under a vacuum. This procedure is not always practical because of the processing and equipment involved. The literature reports that charge-coupled devices (CCDs) give characteristically different responses to different types of radiation. CCDs are small, semiconductor pixel-imaging devices suitable for use in a handheld instrument - they are commonly used in digital cameras. This research explores the potential of employing CCDs for in-situ spectroscopy of $\alpha$ and $\beta$ radiation. This paper focuses on the interactions of $\beta$ particles with CCDs.

As radiation enters a $\mathrm{CCD}$, charge is created through ionization in the active region of the device, as shown in Fig. 1. The evolved charge is then collected by gate electrodes, passed through adjacent pixels, and read out sequentially to form a pixel image based on the location of the created charge within the CCD, and hence that of associated interactions. Different types of artifacts may form in these images, depending on the interactions between the radiation and the CCD. ${ }^{1,2}$ Hypothetically, if radiation scatters through several pixels, this will leave a trail of charge, resulting in a track of pixels. If a large amount of energy is deposited in a given pixel, it may diffuse through the substrate to create large, symmetrical clusters of pixels. If the amount of created charge reaches the capacity of the pixel, it may overflow vertically. This "blooming" would be expected to leave a vertical streak of pixels.

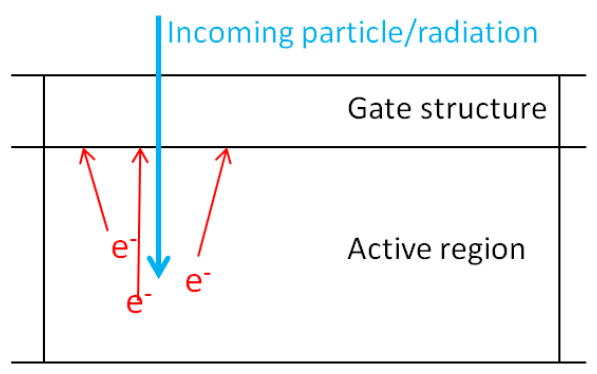

Substrate

Fig. 1. The basic structure of a CCD pixel, showing incoming radiation producing charge, which is collected by the gate structure. 


\section{Prior Art}

Exploratory research at Lancaster University, UK, showed that different types of radiation leave characteristic tracks in CCD images, as shown in Fig. 2. ${ }^{3}$ This phenomenon is consistent with $\alpha$ particles leaving large, symmetrical clusters or long vertical tracks due to blooming, owing to the large amount of charge produced. $\beta$ radiation leaves long curved tracks as each particle scatters through a number of pixels in the $\mathrm{CCD}$, and $\gamma$ radiation leaves small clusters as it passes through relatively unimpeded.
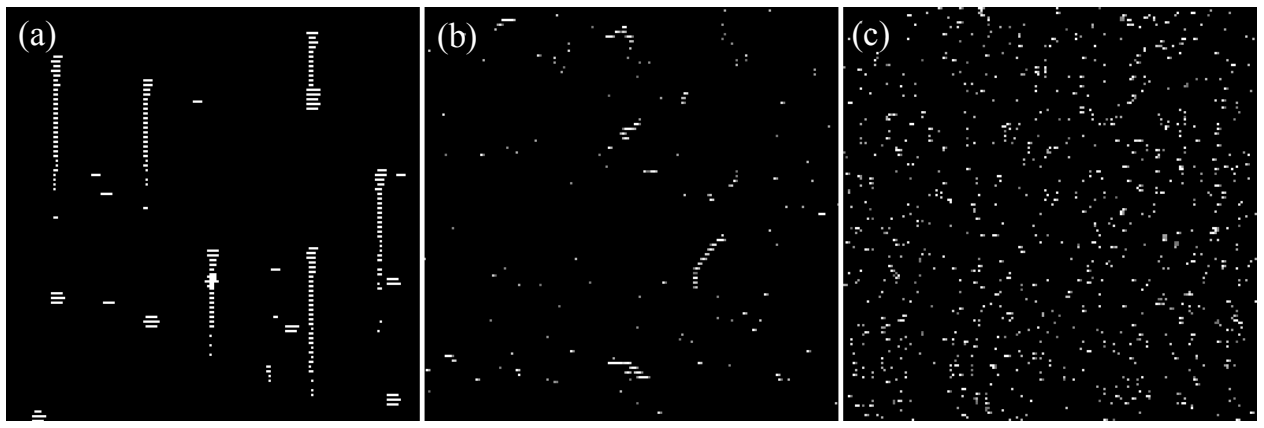

Fig. 2. Previous research showing CCD responses to: (a) $\alpha$ particles $\left({ }^{241} \mathrm{Am}\right)$; (b) $\beta$ particles $\left({ }^{234} \mathrm{Th}\right)$, and (c) low-energy $\gamma$ rays $\left({ }^{241} \mathrm{Am}\right){ }^{3}$

These findings agree with several works that report these differences, ${ }^{2,4,5}$ and it is also observed that $\alpha$-particle cluster sizes increase with increasing $\alpha$-particle energy in a similar silicon-pixel detector. ${ }^{6}$ While these results suggest a potential use of CCD images in spectroscopy, research has focused on astronomical applications and investigating $\mathrm{x}$ rays - for example, where the effects of $\alpha$ and $\beta$ radiation are undesirable. ${ }^{1,7}$ Investigations specifically into $\alpha$ and $\beta$ particle spectroscopy with CCDs have been few.

\section{Investigating $\beta$-Particle Interactions in CCDs}

The CCD used in this research is a Sony ICX825AL, an interline CCD with $1392 \times 1040$ pixels and a pixel size of $6.45 \times 6.45 \mu \mathrm{m}$, for a total imaging area of $6.71 \times 8.98 \mathrm{~mm}$. The imaging area of the CCD is shown in Fig. 3(a), and the CCD connected to electronics in Fig. 3(b). The film covering the imaging area in 3(b) is an aluminized Mylar film that prevents light from entering and interacting with the CCD. This film is $3.0 \mu \mathrm{m}$ thick, consisting of $0.6 \mu \mathrm{m}$ of aluminum and $2.4 \mu \mathrm{m}$ of Mylar. Two layers are applied, reducing the energy of $5.3 \mathrm{MeV}$ incident $\alpha$ particles from a ${ }^{210} \mathrm{Po}$ source by $0.8 \mathrm{MeV}$, calculated using SRIM (Stopping and Range of Ions in Matter). ${ }^{8}$ Therefore $\alpha$ and $\beta$ particles may still be detected. The device is connected to a computer via USB cable. The CCD software bins the pixels in a $2 \times 2$ area. For the rest of this paper, "one pixel" will refer to these $2 \times 2$ pixel bins, such that the total image size is $694 \times 520$ pixels and one pixel is $12.9 \times 12.9 \mu \mathrm{m}$. 


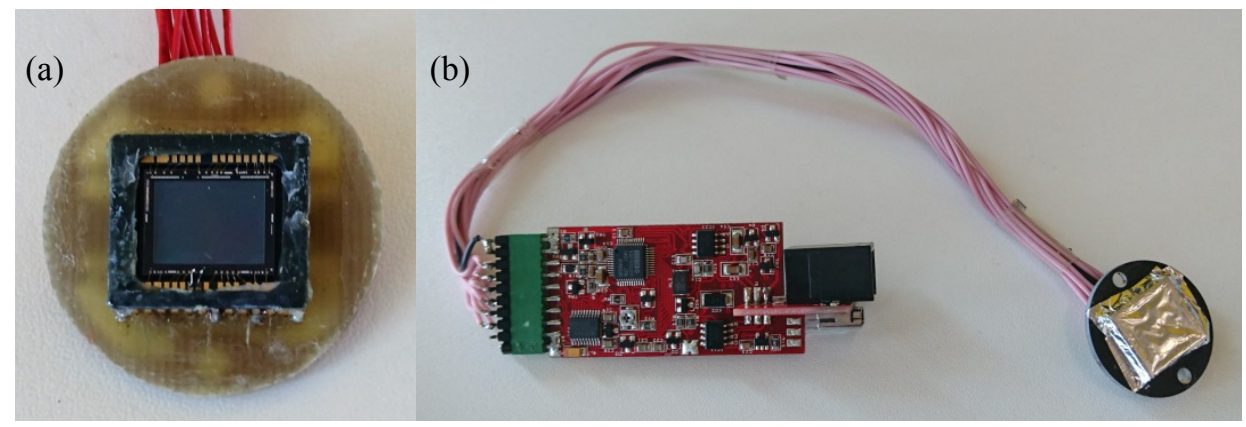

Fig. 3. The CCDs used in this research. (a) Exposed CCD imaging area. (b) CCD with associated electronics and film covering.

Two $\beta$-particle sources were used, ${ }^{60} \mathrm{Co}$ and ${ }^{137} \mathrm{Cs}$. These are $\gamma$ and $\beta$ emitters, with the most common $\beta$ emission having a maximum energy of $310 \mathrm{keV}$ for the ${ }^{60} \mathrm{Co}$ source and $512 \mathrm{keV}$ for the ${ }^{137} \mathrm{Cs}$ source. For this research, modeling and experimentation were used to compare the expected behavior of the $\beta$ radiation to the experimental data. The aim was also to investigate any differences between the two sources which may be due to their difference in energy.

\subsection{Modeling}

Simulations were performed using the Monte Carlo software package CASINO (Monte Carlo Simulation of Electron Trajectory in Solids) ${ }^{9}$ to build a 3D sample through which simulated electrons were passed and their trajectories and energy losses detailed. Preparing the CCD sample was problematic due to insufficient information about the layers within CCD technologies in general. Pixel length and width may be derived from the product datasheet, but layer depths were taken per typical values from several sources $^{1,10,11}$ and the optimal layer depth determined in previous research. ${ }^{3}$ This gave depths for the active layer and a substrate of $60 \mu \mathrm{m}$. The gate structure was not included in the sample, as previous research found negligible effects on the $\beta$ particles. The substrate was simulated to account for any electrons that may be scattered back into the pixel grid. A $10 \times 10$ pixel grid was considered for this sample $(129 \times 129 \mu \mathrm{m})$, with the electrons incident on one pixel in the middle of the grid.

To represent the two sources available for this research, electrons of $310 \mathrm{keV}$ and $512 \mathrm{keV}$ were considered. For each energy, 10,000 electrons were simulated as passing through the sample. A selection of 30 tracks for each electron energy can be seen in Fig. 4. The number of pixels traversed by each track was counted and plotted in a histogram, as shown in Fig. 5. 
(a)

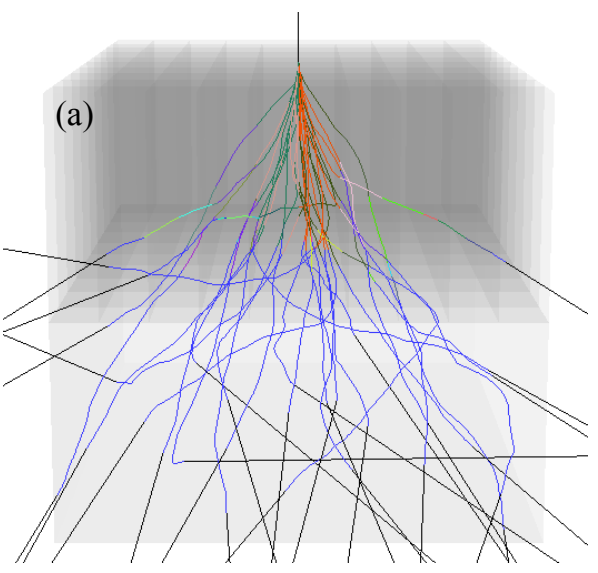

(b)

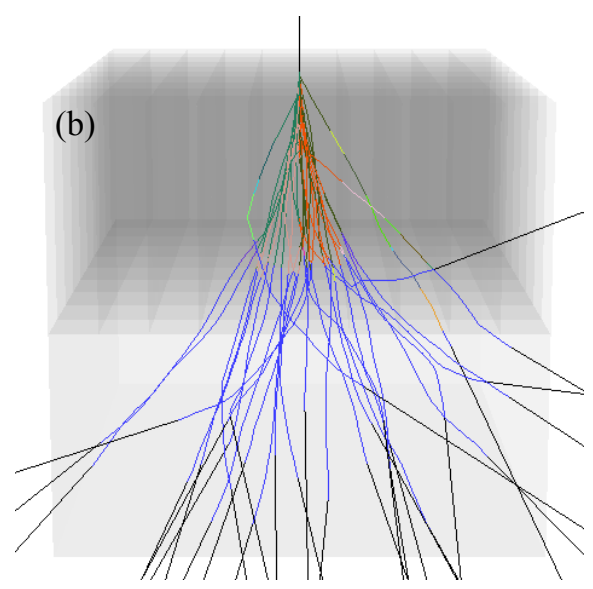

Fig. 4. CASINO simulations showing 30 of the 10,000 tracks for $\beta$ particles with an energy of $310 \mathrm{keV}$ (a) and $512 \mathrm{keV}$ (b) to represent ${ }^{60} \mathrm{Co}$ and ${ }^{137} \mathrm{Cs}$, respectively. $10 \times 10$ binned pixels are simulated. These are $60 \mu \mathrm{m}$ deep on top of a $60 \mu \mathrm{m}$ substrate layer. All regions are silicon. Colors represent different regions the electron has passed through.

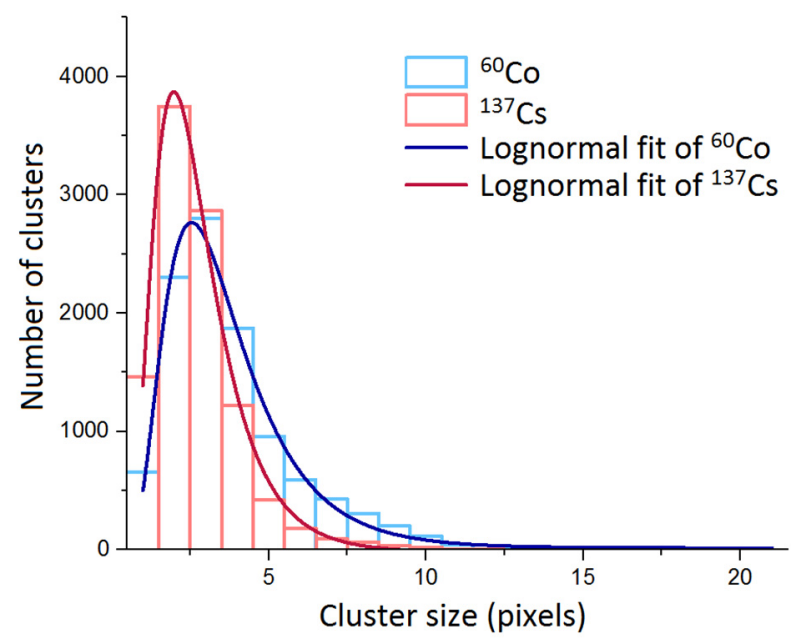

Fig. 5. Histogram of the number of pixels traversed by each track in the CASINO simulations for ${ }^{60} \mathrm{Co}$ $(210 \mathrm{keV})$ and ${ }^{137} \mathrm{Cs}(512 \mathrm{keV})$. Lognormal curves have been fit to each of the data.

In comparing Figs. 4(a) and 4(b), it can be seen that the lower-energy electrons corresponding to ${ }^{60} \mathrm{Co}$ scattered more than higher-energy electrons-as theoretically expected because of the higher scattering cross-section of lower-energy $\beta$ particles. This is depicted in Fig. 5, where the ${ }^{137} \mathrm{Cs} \beta$ particles have a larger peak at a cluster size of two pixels and the ${ }^{60} \mathrm{Co}$ data yield a smaller peak at a larger cluster size (2.5 pixels), with a higher ratio of large clusters. It was found that lognormal curves gave the best empirical fit to these data and were thus used in investigating the experimental data. 


\subsection{Experiment}

The experimental setup is shown in Fig. 6. The CCD was connected by USB to a laptop loaded with the CCD's software, and the ${ }^{60} \mathrm{Co}$ source was placed on top of the CCD, over the imaging area. This procedure was repeated for both sources. A calibration with no source in place was performed for $30 \mathrm{~s}$ to allow the removal of background signals. Exposures were then taken with each source. To reduce the potential of tracks overlapping and being counted as single, exposures were timed to have approximately 1300 clusters - that is, accounting for the different activities of the sources, 5-s exposures were used for ${ }^{60} \mathrm{Co}$ and 1 -s exposures were used for ${ }^{137} \mathrm{Cs}$.

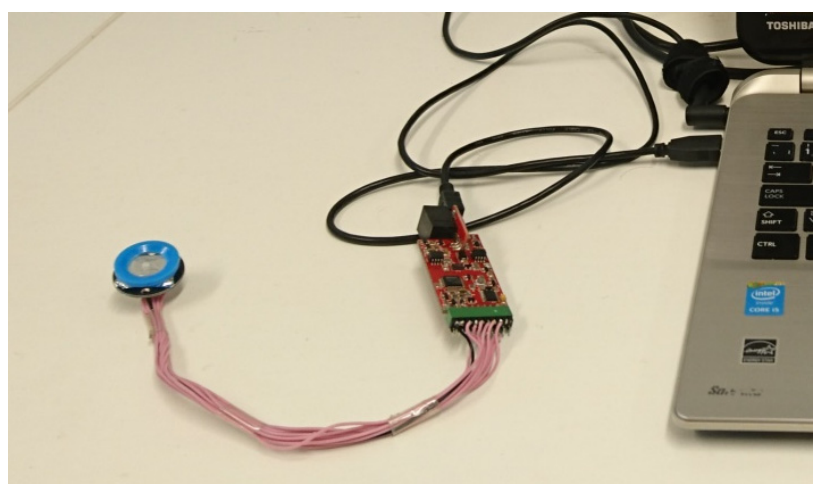

Fig. 6. The experimental setup, showing the ${ }^{60} \mathrm{Co}$ source (the disc with the blue ring) in contact with the CCD, which was connected to a laptop containing the imaging software used in this work.

Twenty exposures were taken for each source, yielding total clusters of 26,003 and 28,056 for Co and Cs, respectively. Example sections for one exposure to each source are provided in Fig. 7.
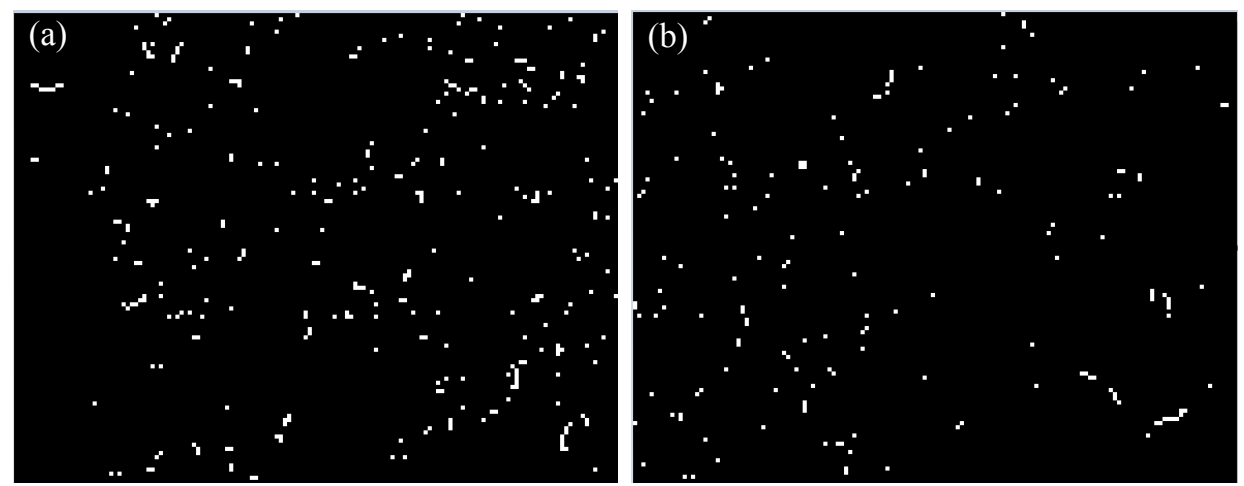

Fig. 7. Example image sections from one exposure to: (a) ${ }^{60} \mathrm{Co}(5 \mathrm{~s})$, and (b) ${ }^{137} \mathrm{Cs}(1 \mathrm{~s})$. The sections are $146 \times 114$ pixels and representative of all exposures. 
Using MATLAB, clusters were identified as any pixels connected horizontally, vertically, or diagonally. The total number of pixels in each track was counted and normalized as a percentage of the total number of clusters. This figure was plotted in a histogram, as shown in Fig. 8.

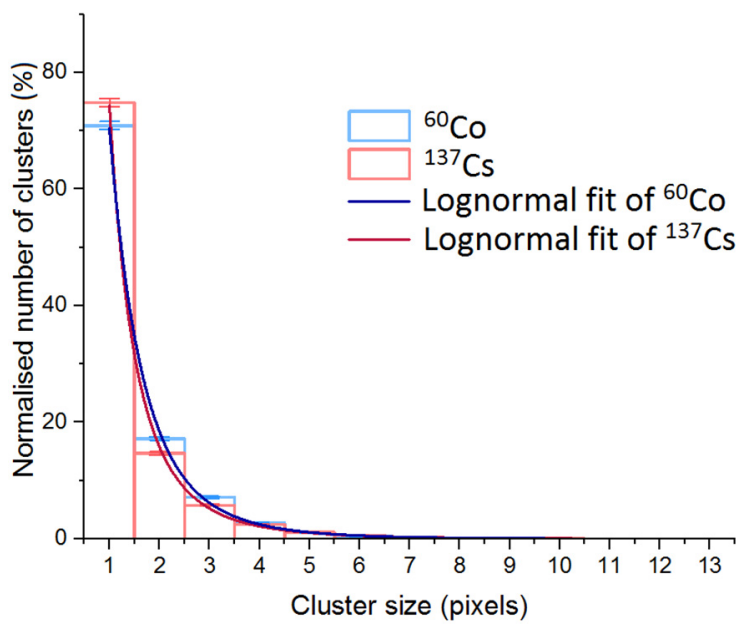

Fig. 8. Histogram of cluster sizes produced in the 20 exposures of the CCD to each source. The data are normalized to a percentage of the total number of clusters. Lognormal curves were used to fit the data.

It is evident that the peaks of the data are not entirely consistent with those in the simulations. For both sources, the experimental peak is at one pixel. These experimental images contain clusters from $\gamma$ radiation, and it is hypothesized that they are responsible for the shift to smaller clusters, since $\gamma$ radiation typically produces small, one- or twopixel clusters. The lognormal curves applied to these data are also very similar, possibly owing to the small energy difference between the sources that were used. The trends identified in the simulations are evident; however, the ${ }^{137} \mathrm{Cs}$ data have a larger peak, while the ${ }^{60} \mathrm{Co}$ exhibits a larger proportion of large clusters.

Another difference is that the cluster sizes are generally smaller than expected from the simulations, with the curves approaching zero at around seven pixels in the experiment, compared with 10-12 pixels in the simulations. This discrepancy is most likely due to the threshold set by the software to reduce noise. Every pixel the electrons passed through in the simulations was counted as part of the track, whereas, experimentally, if only a small amount of charge is produced in a given pixel, it is considered empty. Thus the tracks may be split or simply reduced in size if some pixels fall below the threshold.

\section{Investigating $\alpha$ Particle Interactions in CCDs}

Research investigating the response of the CCDs to $\alpha$-particles was also performed, using a ${ }^{210} \mathrm{Po}$ source chosen as a pure $\alpha$-particle emitter that produces $\alpha$-particles of a single 
energy. The CCD images produced contain horizontal streaks, as shown in Fig. 9, in contrast to the vertical streaks in Fig. 2(a). This is presumed to be due to an overflow of charge at the output amplifier into that from the subsequent pixel to be read out, rather than a vertical overflow at the pixel site.

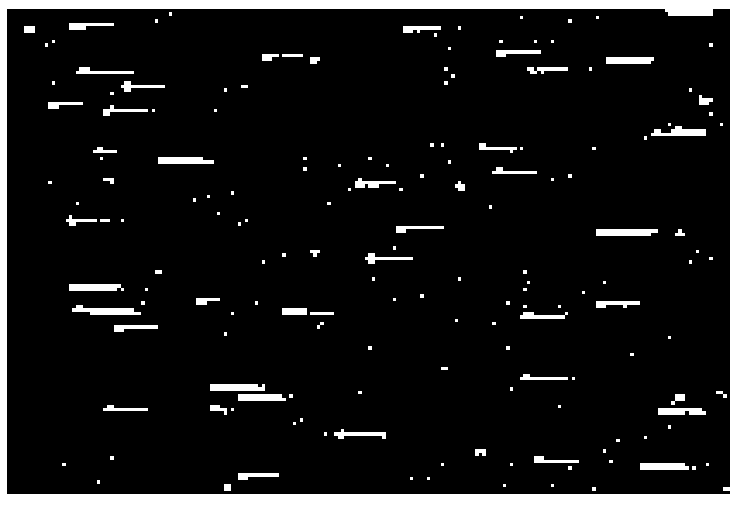

Fig. 9. Section of an image produced by a $30 \mathrm{~s}$ exposure of the CCD in contact with a ${ }^{210}$ Po source.

Ten images were produced, each with a $30 \mathrm{~s}$ exposure to the ${ }^{210} \mathrm{Po}$ source, and the data summed to give the total number of streaks of different lengths, as shown in Fig. 10. Pixel clusters were considered streaks only if they were more than six pixels in length, giving a total streak number of 1625 . Some of the streaks have small gaps where the charge dropped below the threshold set by the software. Where there was a single pixel gap between two streaks, it was assumed they were part of the same streak, and the length was calculated to include both parts. A Gaussian curve was fit to the data using MATLAB to give the mean length of the streaks as 14 pixels.

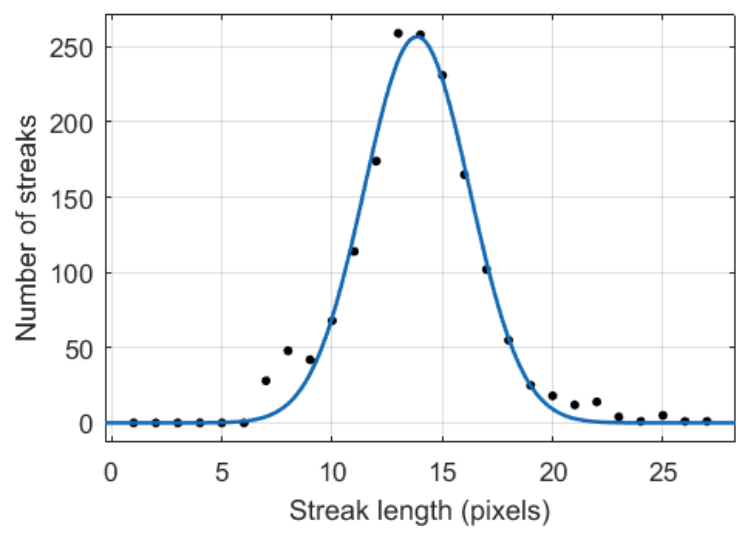

Fig. 10. Graph showing total streaks of different lengths (black dots). A Gaussian curve has been fit to the data (blue line) giving a mean length of 14 pixels. 


\section{Future Work}

A wider range of $\beta$-particle energies will be investigated in future work to check whether the trends identified are more significant over a wider range in energy. CASINO simulations will estimate the energy deposited in each pixel and will be compared with the software threshold for a more realistic view on the expected lengths of $\beta$-particle tracks. New exposures will be taken of the sources, with the $\beta$ radiation blocked by thin aluminum that has minimal effect on $\gamma$ radiation, to confirm or deny hypotheses on the differences between the simulation and experiment. Using all collected data, researchers will attempt to separate $\beta$ and $\gamma$ peaks from the mixed source.

Research into $\alpha$-particle interactions will continue using an accelerator to obtain $\alpha$ particles of various energies. This will allow further exploration of the potential use of CCD devices for $\alpha$-particle spectroscopy, as it is expected that streak length will vary with $\alpha$-particle energy.

\section{Summary}

Charge-coupled devices show significant potential for the spectroscopy of $\alpha$ and $\beta$ radiation. Artifacts in the images produced under exposure to $\beta$ radiation have been investigated; Monte Carlo simulations find that the lower-energy $\beta$ particles leave longer tracks in CCDs, consistent with their higher scattering cross-section. While this is observed with a small difference between the ${ }^{60} \mathrm{Co}$ and ${ }^{137} \mathrm{Cs}$ sources experimentally, the distinction is more pronounced in simulations. The differences between simulation and experiment may lie in two causes: the influence of $\gamma$ radiation, which creates small 1-2 pixel clusters for both sources, and the CCD threshold software's removal of pixels with only a small charge, thereby splitting tracks in two and reducing the overall size of pixel clusters. Further investigations will be carried out on $\beta$-particle sources with $\beta$-particle energies that are more significantly different to confirm whether these trends are consistent over a wider energy range.

Images produced under exposure to a ${ }^{210} \mathrm{Po} \alpha$-particle source show horizontal streaks averaging 14 pixels in length. This average may vary with $\alpha$-particle energy, a question that will be resolved experimentally using an accelerator to obtain a variety of $\alpha$-particle energies.

\section{Acknowledgments}

This research was supported by the Next Generation Nuclear Centre for Doctoral Training, the Engineering and Physical Sciences Research Council (EPSRC), and the Lloyd's Register Foundation. M. J. Joyce acknowledges the support of the Royal Society through a Wolfson Research Merit Award. 


\section{References}

1. A. Aguilar-Arevalo et al., J. Instrum. 10, P08014 (2015).

2. J. Estrada, J. Molina, J. J. Blostein, and G. Fernández, Nucl. Instrum. Meth. Phys. Res. Sect. A 665, 90 (2011).

3. R. Newton, J. Dykes, M. J. Scott, and M. J. Joyce, Alpha Contamination Assay, Dosimetry and Spectrometry Using Charge Coupled Devices, in IEEE Nuclear Science Symposium \& Medical Imaging Conference (2016).

4. T. Saad Saoud et al., IEEE Trans. Nucl. Sci. 61, 3380 (2014).

5. T. S. Lomheim et al., IEEE Trans. Nucl. Sci. 37 (6-1), 1876 (1990).

6. M. Campbell et al., Nucl. Instrum. Meth. Phys. Res. A 591, 1 (2007).

7. A. R. Smith et al., Radiation events in astronomical $\{C C D\}$ images, in Proc. SPIE 4669, 172 (2002).

8. J. F. Ziegler et al., Nucl. Instrum. Meth. Phys. Res. B 268, 1818 (2010).

9. H. Demers et al., Scanning 33, 135 (2011).

10. F. Li and A. Nathan, CCD Image Sensors in Deep-Ultraviolet: Degradation Behavior and Damage Mechanisms (Springer Science \& Business Media, 2006).

11. B. Burke, P. Jorden, and V. U. Paul, Exp. Astron. 19, 69 (2006). 